\title{
Interference Cancellation and Performance Enhancement in Coexistent Heterogeneous Wireless Packet Networks using Packet Transmission Time Sharing
}

\author{
G.M.Tamilselvan, Member, IACSIT, Dr.A.Shanmugam
}

\begin{abstract}
In ubiquitous networking environments, we generally need two or more heterogeneous communication systems coexisting in a single place. Especially, wireless local area networks (WLANs) based on IEEE 802.11 specifications and wireless personal area networks (WPANs) based on IEEE 802.15.4 specifications need to coexist in the same Industrial, Scientific and Medial (ISM) band. If the WPAN communication coverage is expanded using a cluster-tree network topology, then the 802.15.4 network is more susceptible to interference from neighboring WLANs. In this paper, we propose packet transmission management (PTM) scheme for mitigating the interference effects in WPAN. To evaluate the performance of the proposed scheme, the performance metrics such as bit error, throughput, average end-end delay and average jitter is measured using Qualnet 4.5 simulator. The measurement result shows that the proposed scheme is effective in an IEEE 802.15.4 network for various topologies in the presence of multiple IEEE 802.11 interferers.
\end{abstract}

Index Terms-Coexistence, Heterogeneous wireless network, IEEE 802.15.4, IEEE 802.11b, Packet Transmission Management.

\section{INTRODUCTION}

Ubiquitous networking enables many objects or devices to be connected and communicate with each other. Wireless local area networks (WLANs) based on IEEE 802.11b and wireless personal area networks (WPANs) based on IEEE 802.15.4 can play an important role in future ubiquitous networks. Both networks share the same $2.4 \mathrm{GHz}$ Industrial, Scientific and Medical (ISM) band.

ISM band is widely used among popular wireless network standards such as IEEE 802.15.4 Low-Rate Wireless Personal Area Network (LRWPAN), IEEE 802.11b Wireless Local Area Network (WLAN), IEEE 802.15.3, and Bluetooth. Because of the mobility and ubiquitous deployment of wireless systems, there are many scenarios where different systems operate in the same place at the same time. Hand-held PDA can use a Bluetooth device to connect to a laptop with $802.11 \mathrm{~b}$ WLAN. The ISM band is also used by home appliances such as microwave ovens. The microwave oven in the house can be turned on when

Manuscript Received June, 2009

G.M.Tamilselvan is with the Department of Electronics and Communication Bannariamman Institute of Technology, Sathyamangalam638401

Erode dt, Tamilnadu, India. (email:tamiltamil@rediffmail.com)

Dr.A.Shanmugam is with the Department of Electronics and Communication Bannariamman Institute of Technology, Sathyamangalam, Erode dt-638401, Tamilnadu, India (email:dras@yahoo.co.in) cordless phone is being used.

Coexistence is defined as "the ability of one system to perform a task in a given shared environment where other systems may or may not be using the same set of rules" .Especially, for mission-critical applications using wireless systems; the coexistence becomes a top priority issue in system design. For example, if 802.15.4 sensor network system is to be deployed in the hospital building for emergency medical care, a main design issue will be providing the coexistence of 802.15.4 and other wireless systems. In case the other system causes radio channel interference, the sensor network system can not continue the normal operation and may lose critical information such as emergency patient vital signals and emergency patient information.

Growing concern is in the coexistence of 802.1.5.4 and 802.11 b. There are many practical situations and scenarios where 802.15 .4 and 802.11 b systems operate simultaneously. An interesting system is sensor network system employing IEEE 802.15.4(WPAN) technology. Recently, Wireless personal area networks (WPAN) are one of most essential technologies for implementing ubiquitous computing. Wireless personal area networks are used to convey information over relatively short distances. Unlike wireless local area networks, connections effected via WPANs involve little or no infrastructure. This feature allows small, power-efficient, inexpensive solutions to be implemented for a wide range of devices.

The IEEE 802.15.4 would be widely adopted for various applications such as detection, remote control, tracking, and monitoring. The scope of IEEE 802.15.4 [1][2] is to define the physical layer (PHY) and medium access control (MAC) sub layer specifications for low data rate wireless connectivity with fixed, portable, and moving devices with no battery or very limited battery consumption requirements typically operating in the personal operating space (POS) of $10 \mathrm{~m}$. It is foreseen that, depending on the application, a longer range at a lower data rate may be an acceptable tradeoff. The purpose of IEEE 802.15.4 is to provide a standard for ultra-low complexity, ultra-low cost, ultra-low power consumption, and low data rate wireless connectivity among inexpensive devices.

Many practical wireless sensor network systems cover a large area of interest. The examples include wild life habitat monitoring, hospital emergency medial care and health monitoring, forest fire detection and tracking, traffic monitoring and others. Because those systems have a large 
coverage area and the same area can have other human activities such as residences and leisure activities, it is reasonably assumed that the 802.15.4 system will be operating with other systems. It is interesting to note that the effect caused by radio interference is not reciprocal when multiple wireless systems operate simultaneously. It is because of the difference in radio transmission range. 802.11 b uses a longer range radio than 802.15.4 system. 802.11b WLAN has radio range of $100 \mathrm{~m}$ and 802.15.4 LRPAN has radio range of $10 \mathrm{~m}$ [10]. Thus, $802.11 \mathrm{~b}$ can give radio interference to 802.15 .4 system in a large area and from a long distance. Therefore, large-scale 802.15.4 based sensor network system is vulnerable to the interference from 802.11 b. Moreover, 802.11 b systems are employed in many portable devices including hand-held Personal Data Assistant (PDA) and laptop computers. Due to the omnipresence and mobility of those systems, there is a high chance of operating 802.15.4 and 802.11b in the same environment. There are many situations, where 802.15.4 and $802.11 \mathrm{~b}$ need operate in the same system. For example, 802.15.4 wireless sensor nodes forward the sensing data to a laptop, which will be send the collected data over $802.11 \mathrm{~b}$ WLAN to the central computer for processing and further analysis.

In the coexistence of IEEE 802.15.4 and IEEE 802.11b, the main concern is the performance degradation of IEEE 802.1.5.4 caused by the interference of IEEE 802.11b. A measurement study reported that over $92 \%$ of the 802.15.4 frames were lost by the interference of IEEE 802.11b [11]. In this paper, we propose a scheme using packet transmission management to solve the performance degradation of IEEE 802.15.4. Especially, the proposed scheme is intended to support coexistence performance issue for IEEE 802.15.4 multi-hop network.

The rest of this paper is organized as follows: Section 2 summarizes the related works. The proposed scheme is presented in Section 3. Simulation results are discussed in Section 4. Finally, we conclude our paper.

\section{RELATED WORK}

Figure 1 shows the operational frequency spectrum of both IEEE 802.15.4(ZigBee) and IEEE 802.11b (WLAN) networks. A WLAN system has eleven channels. Each channel occupies $22 \mathrm{MHz}$ and up to 3 separate channels can be simultaneously used without any mutual interference. Channels 1, 6, and 11 can be used for neighboring IEEE 802.11 WLAN Access Points (APs), as shown in Figure 1, to mitigate the interference. On the other hand, ZigBee networks have sixteen channels in $2.4 \mathrm{GHz}$ band which can be used simultaneously without any mutual interference among them. Since the transmission power of WLAN is usually 100 times larger than that of ZigBee networks, we focus on the effect of interference from WLAN to ZigBee and the interference among WLAN nodes because of collision caused by multiple transmissions.

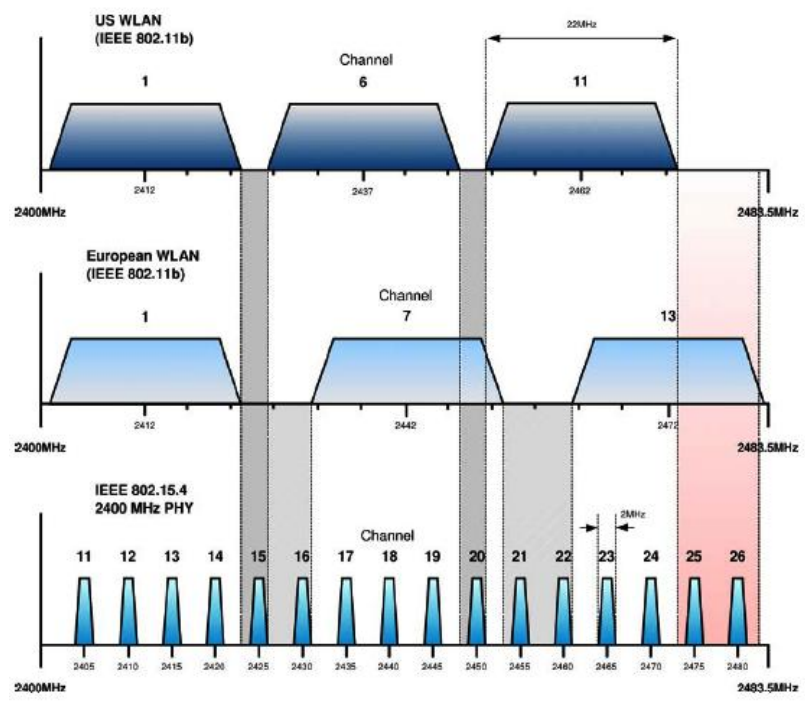

Figure.1. Frequency Spectrum of ZigBee and WLAN Networks

In IEEE 802.15.4 standard, a transmission between PAN coordinator and devices is performed inside the $2.4 \mathrm{GHz}$ ISM band, at $250 \mathrm{kbps}$, and exploiting one of the 16 available channels. As shown in table 1, such channels have a $2 \mathrm{MHz}$ bandwidth and are uniformly distributed within the ISM band.

TABLE 1. 2.4GHZ ISM B AND, IEEE 802.15.4 AND IEEE 802.11 CHANNELS

\begin{tabular}{|c|c|c|c|c|}
\hline & \multicolumn{2}{|c|}{ IEEE 802.11b } & \multicolumn{2}{|c|}{ IEEE 802.15.4 } \\
\hline & Ch. & Freq. (GHz) & Ch & Freq. (GHz) \\
\hline \multirow{16}{*}{$\begin{array}{c}2.4 \mathrm{GHz} \\
\text { ISM } \\
\text { Band }\end{array}$} & 1 & $2.401-2.423$ & 1 & 2.405 \\
\hline & 2 & $2.404-2.426$ & 2 & 2.410 \\
\hline & 3 & $2.411-2.433$ & 3 & 2.415 \\
\hline & 4 & $2.416-2.438$ & 4 & 2.420 \\
\hline & 5 & $2.421-2.443$ & 5 & 2.425 \\
\hline & 6 & $2.426-2.428$ & 6 & 2.430 \\
\hline & 7 & $2.431-2.453$ & 7 & 2.435 \\
\hline & 8 & $2.436-2.458$ & 8 & 2.440 \\
\hline & 9 & $2.441-2.463$ & 9 & 2445 \\
\hline & 10 & $2.446-2.468$ & 10 & 2450 \\
\hline & 11 & $2.451-2.473$ & 11 & 2455 \\
\hline & 12 & & 12 & 2460 \\
\hline & 13 & & 13 & 2465 \\
\hline & 14 & & 14 & 2470 \\
\hline & & & 15 & 2475 \\
\hline & & & 16 & 2480 \\
\hline
\end{tabular}

Some related researches study the coexistence problem between the IEEE 802.15.4 and the 802.11b [3], [4].In [3], the packet error rate (PER) of the IEEE 802.15.4 under the IEEE $802.11 \mathrm{~b}$ and IEEE 802.15.1 is obtained by experiments only. In [4], the impact of an IEEE 802.15.4 network on the IEEE $802.11 \mathrm{~b}$ devices is analyzed. Channel Conflict Probabilities between IEEE 802.15 based Wireless Personal Area Networks is modeled in [5]. Packet Error Rate of IEEE 802.15.4 under IEEE 802.11b interference is 
analyzed in [6].In [7] Packet Error Rate of IEEE 802.11b under IEEE 802.15.4 interference is analyzed. In [8] channel conflict probabilities between IEEE $802.11 \mathrm{~b}$ and IEEE 802.15.4 have been modeled. In [9] channel collision between IEEE 802.15.4 and IEEE 802.11b for circular and grid topology is analyzed with the mobility model. To the best knowledge of the authors, performance enhancement of coexistence heterogeneous network for circular, grid and random topology by packet transmission management has not been discussed in the literature.

\section{PROPOSED SCHEME}

In this paper, we propose a packet transmission based analysis for the performance metrics such as bit error, throughput, average End-End delay and average jitter of IEEE 802.15.4. We consider a heterogeneous network with circular, grid and random topology.

Here the performance of IEEE 802.15.4 under the interference of IEEE $802.11 \mathrm{~b}$ and the interference among WLAN nodes because of multiple transmissions is analyzed using Qualnet 4.5 simulation. For simulation, the slotted CSMA/CA of the IEEE 802.15.4 model is developed using Qualnet 4.5.The scenario of coexistence heterogeneous network for circular, grid and random topology is shown in figure 2(a-c).

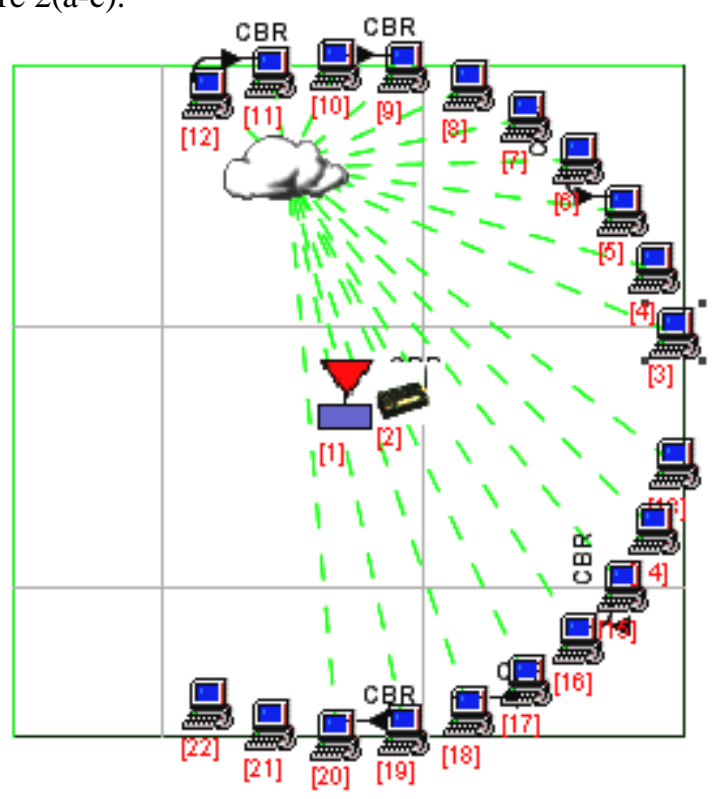

Figure 2.a Coexistence Heterogeneous network scenario for Circular Topology

The figure 2.a shows the scenario of Coexistence Heterogeneous network scenario for Circular Topology developed in Qualnet 4.5 simulator. In this scenario 2 WPAN nodes and 20 WLAN nodes are used. The node 1 is an End device sending packets to the PAN coordinator which is numbered as 2.The WLAN nodes are placed at equal distance about $5 \mathrm{~m}$ from the PAN coordinator. From 20 WLAN nodes 5 nodes are assumed as transmitting nodes. Multiple transmissions in WLAN cause collision which severely affects the performance of WPAN node. The node 1 is RFD (Reduced Functional Device) and node 2 is FFD (Fully Functional Device). The figure 2.b shows the Qualnet scenario for Grid topology with 2 WPAN nodes and 20 WLAN nodes. In grid topology nodes are placed at equal distance from one another .The distance between 2 nodes are fixed as $2 \mathrm{~m}$.

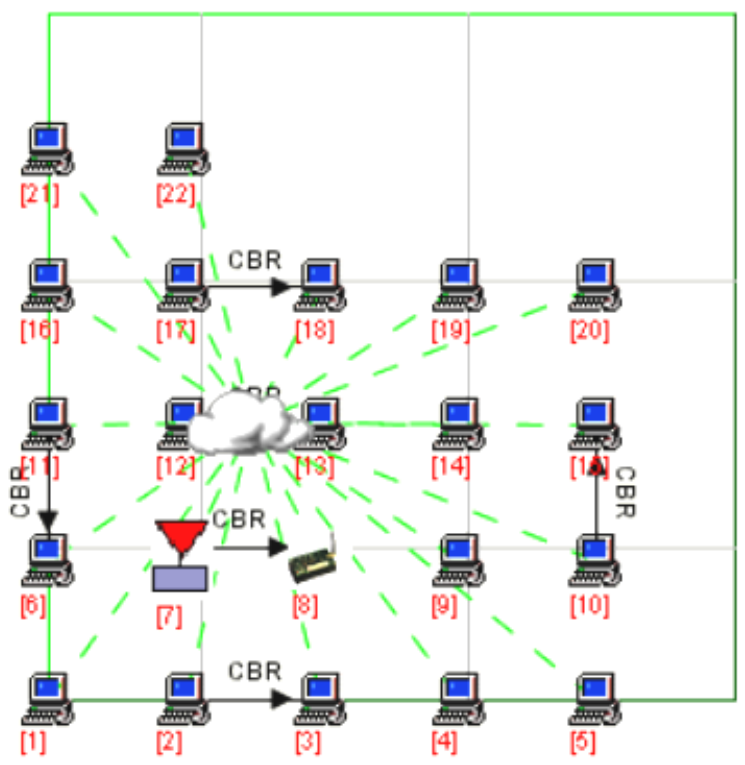

Figure 2.b Coexistence Heterogeneous network scenario for Grid Topology.

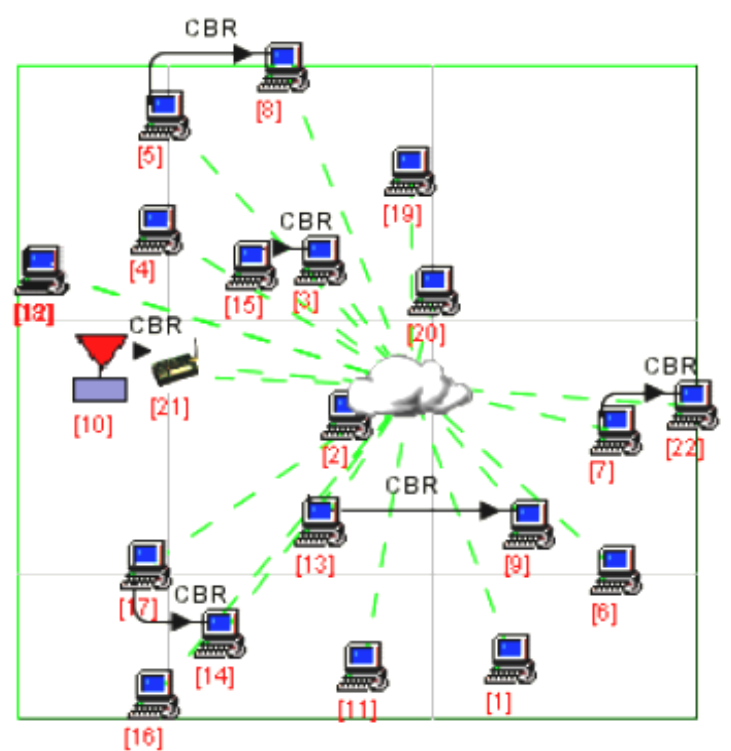

Figure 2.c Coexistence Heterogeneous network scenario for Random Topology

The figure 2.c shows the Qualnet scenario for Random topology with 2 WPAN nodes and 20 WLAN nodes. For this topology seed value is taken as 5.All the nodes are placed randomly except the WPAN nodes. In random topology the distance between the WPAN nodes are fixed as $1 \mathrm{~m}$. PTM scheme is compatible with TDMA because each transmitting node in the scenario is allotted unique transmission time.

The PHY of the IEEE 802.15.4 at $2.4 \mathrm{GHz}$ uses offset quadrature phase shift keying (OQPSK) modulation. Denote that the $E_{b} / N_{0}$ is the ratio of the average energy per information bit to the noise power spectral density at the receiver input, in the case of an additive white Gaussian noise (AWGN) channel. Then the bit error rate (BER), $P_{B}$, can be expressed as 


$$
P_{B}=Q\left(\sqrt{\frac{2 E_{b}}{N_{0}}}\right)
$$

Where $\mathrm{Q}(\mathrm{x})$ is

$$
Q(x)=\frac{1}{\sqrt{x}} \int_{x}^{\infty} \exp \left(\frac{-u^{2}}{2}\right) d u
$$

Figure 3 shows the relationship between the bit error rate and $E_{b} / N_{o}$ simulated in Matlab.

The bit error rate decreases when $E_{b} / N_{o}$ increases. The noise power spectral density increases when collision increases. As the number of WLAN sources increases, the BER of IEEE 802.15.4 increases because contentions among multiple WLANs increase the channel usage and cause collisions, which is more powerful interference, source to.

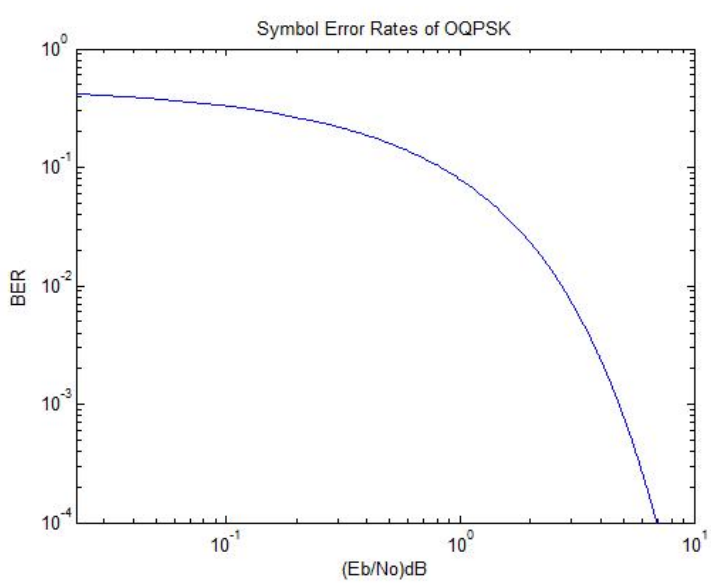

Figure 3: Theoretic Bit Error Rate of OQPSK

\section{Simulation Results AND Discussion}

To evaluate the effectiveness of the proposed scheme in a coexistence heterogeneous wireless network, a simulation study was conducted using Qualnet 4.5 simulator. The simulation configuration and parameters used in this paper is shown in Table 2 .

To study the impact of coexistence on the performance of the 802.15.4 network, measurements were made in a simple experimental environment as shown in Fig.2(a-c). In all mentioned topologies 25\% WLAN nodes are transmitting nodes. The effectiveness of the proposed scheme was measured with four different metrics: Bit error, Throughput, Average End-End delay and Average jitter.

TABle 2. Simulation CONFIGURATION AND PARAMETERS

\begin{tabular}{|c|c|c|}
\hline Parameter & IEEE 802.11b & IEEE 802.15.4 \\
\hline Number of Nodes & 20 & 2 \\
\hline Transmission Power & $20 \mathrm{dbm}$ & $3 \mathrm{dbm}$ \\
\hline Modulation & CCK & OQPSK \\
\hline MAC Protocol & 802.11 & 802.15 .4 \\
\hline Routing Protocol & Bellman ford & AODV \\
\hline No of Packets & 100 & 100 \\
\hline Payload Size & $1500 \mathrm{bytes}$ & $105 \mathrm{bytes}$ \\
\hline Simulation Time & \multicolumn{2}{|c}{$30 \mathrm{~s}$} \\
\hline Packet Interval & $1 \mathrm{~ms}$ & $1 \mathrm{~ms}$ \\
\hline
\end{tabular}

\begin{tabular}{|c|c|c|}
\hline Packet Transmission Time & $1 \mathrm{~s}$ & $1 \mathrm{~s}$ \\
\hline Test bed size & \multicolumn{2}{|c|}{$10 \mathrm{~m} \times 10 \mathrm{~m}$} \\
\hline Topology & Circular, Grid and Random \\
\hline
\end{tabular}

The figure 4.(a-d) shows the performance of 802.15.4 network and other WLAN nodes for the four different metrics by managing the packet transmission time.

The bit error detail of the received data for circular topology is shown in figure 4.a.In circular topology node1 and 2 are IEEE 802.15.4 nodes and other nodes are IEEE 802.11b Nodes. When the PTM (Packet Transmission Management) is adapted for circular topology the bit error of IEEE 802.15.4 becomes zero. The measurement results are analyzed for mobility model also. The bit error of other WLAN nodes is also reduced to zero because of the proposed scheme. Because of this packet transmission management collision free transmission is realized among WLAN nodes. Normally the collision among WLAN nodes causes serious interference in WPAN node because of multiple transmissions.

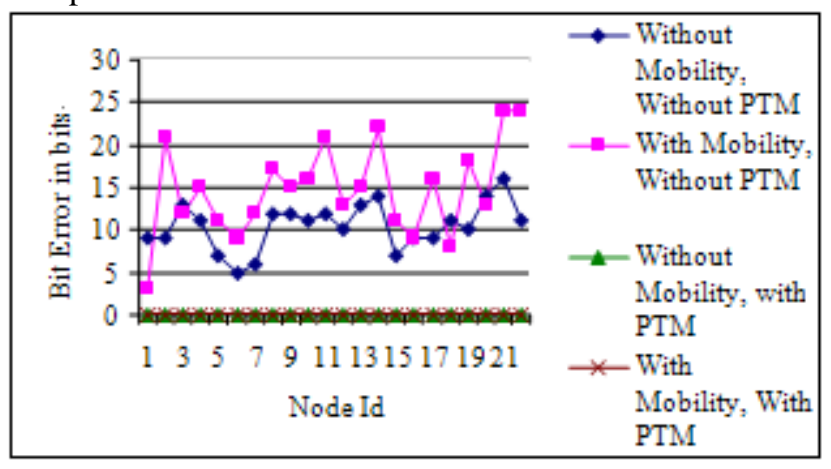

Figure 4.a Bit Error analysis for Circular Topology

The figure 4.b shows the throughput for Circular Topology by varying the packet transmission time. After the implementation of PTM scheme the throughput of all the nodes are increased considerably. For the circular topology the throughput value is measured with and without the mobility model. The collision free transmission results in higher throughput for WPAN and WLAN nodes. In this packet transmission management throughput of the PAN coordinator increased by 7.64 times because of error free transmission. The throughput of WLAN node is increased by 105.6 times.

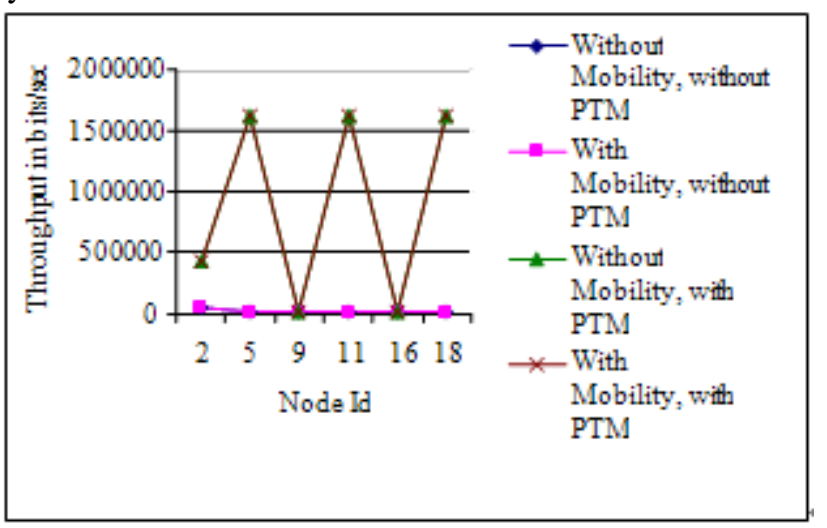

Figure 4.b Throughput analysis for Circular Topology

The average end-end delay of WPAN and WLAN nodes is shown in figure 4.c.The simulation is done with the 
mobility model by fixing the speed about $10 \mathrm{~m} / \mathrm{s}$. The average end-end delay of the WPAN node (node id is marked as 2) after the packet transmission management is reduced 19.5 times as before the packet transmission management is adapted.

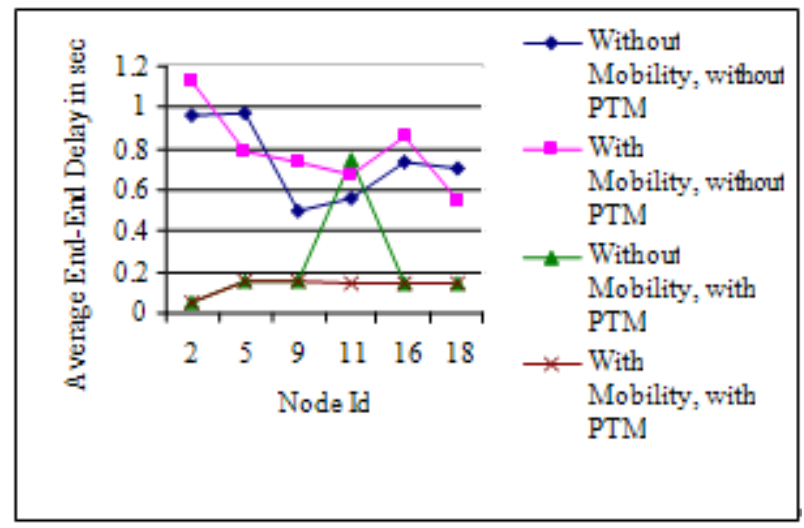

Figure 4.c Average End-End delay analysis for Circular Topology

The average jitter is shown in figure 4.d. The average jitter value is maximum when the transmission time management is not adapted. The simulation result shows that the average jitter is reduced by 16.6 times for WPAN node (node id 2) and for WLAN (node id 5) is reduced by 7 times after adapting the proposed scheme.

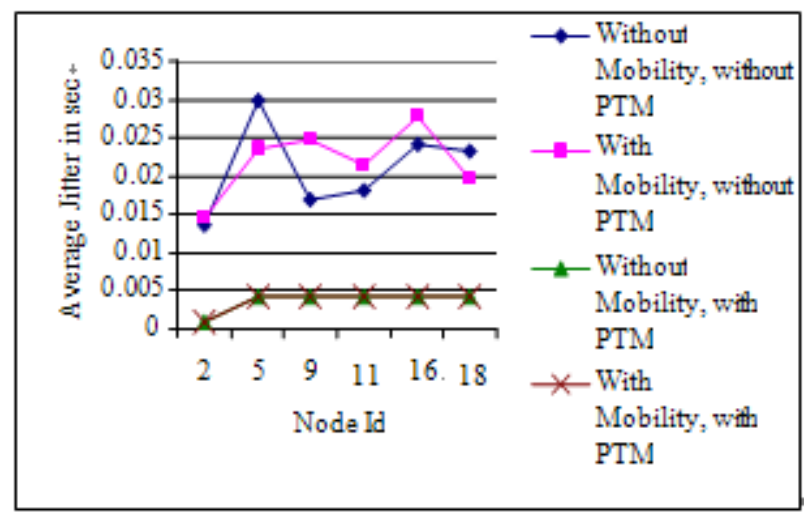

Figure 4.d Average Jitter analysis for Circular Topology

The figure 5.(a-d) shows the performance analysis of 802.15.4 network and other WLAN nodes for grid topology. The grid topology scenario is shown in figure 2.b.The simulation study is conducted with and without the mobility model. In grid topology node 7 and 8 are end device and PAN coordinator respectively. Rest of the nodes is WLAN nodes. Among the 20 WLAN nodes 2,10,11,12 and 17 are transmitting nodes.

The bit error analysis for grid topology is shown in figure 5.a.The bit error becomes zero when the PTM scheme is used in each transmission. The impact of WPAN interference on WLAN nodes is negligible because of the less power spectral density. But the impact of WLAN interference on WPAN nodes is results in significant performance degradation of WLAN nodes. We can analyze the bit error for both static and mobility network from the figure. The bit error is less for WPAN node than for WLAN node.

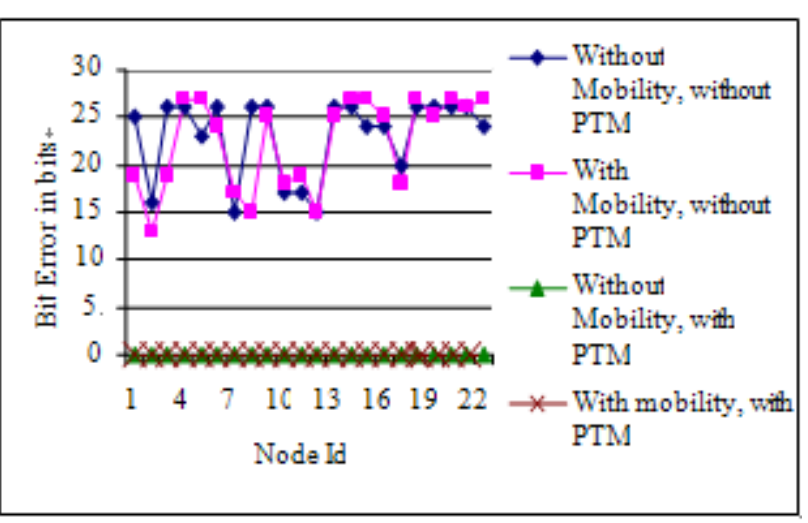

Figure 5.a Bit Error analysis for Grid Topology

The figure 5.b shows the throughput analysis for grid Topology by varying the packet transmission time. After the implementation of PTM scheme the throughput of all the nodes are increased considerably. As like circular topology the throughput value is measured with and without the mobility model for grid topology also. The collision free transmission results in higher throughput for WPAN and WLAN nodes. In this packet transmission management throughput of the PAN coordinator increased by 7 times because of error free transmission. The throughput of WLAN node is increased by 106.4 times.

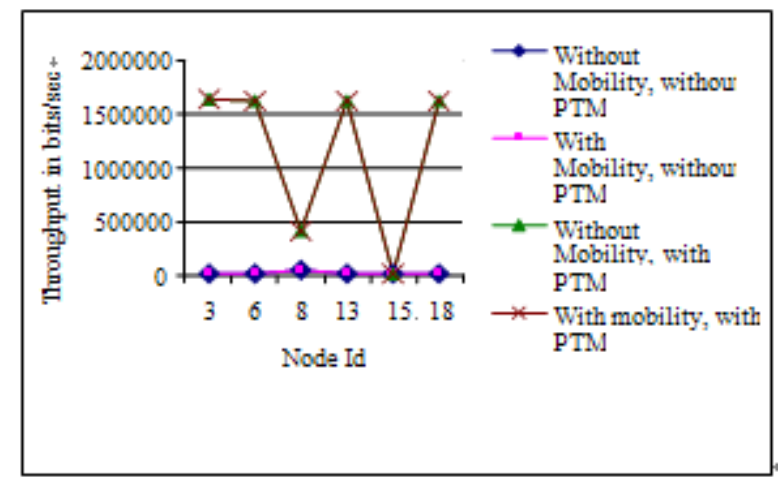

Figure 5.b Throughput analysis for Grid Topology

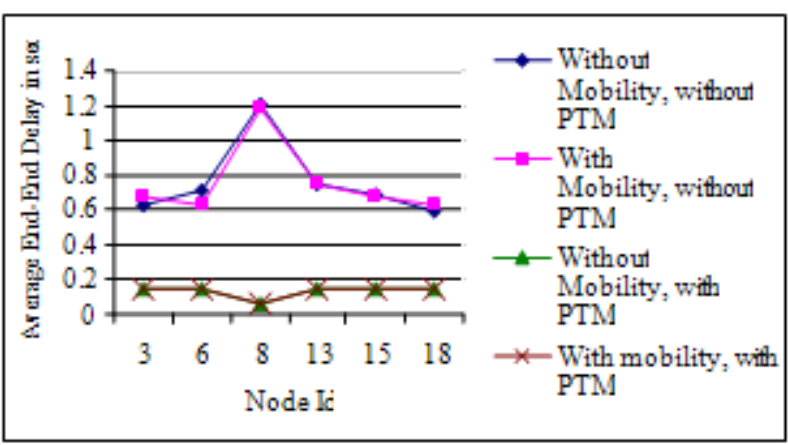

Figure 5.c Average End-End delay analysis for Grid Topology.

The figure 5.c shows the average end-end delay analysis for grid topology with 22 heterogeneous wireless nodes. Due to the application of PTM scheme the end-end delay is decreased 18.8 times for WPAN node when the node is stable and the end-end delay decreased 18.6 times when the node is moving randomly. For the WLAN nodes (examplenode 13 ) the end-end delay is reduced 5 times when node is stable as well as moving randomly with the speed of $10 \mathrm{~m} / \mathrm{s}$.

The average jitter is shown in figure 5.d.The average jitter 
value for WPAN node is minimum when compared with the WLAN node for both the cases before and after adapting the PTM scheme. The average jitter value is 15 times reduced after the proposed scheme is adapted for packet transmission in both the cases.

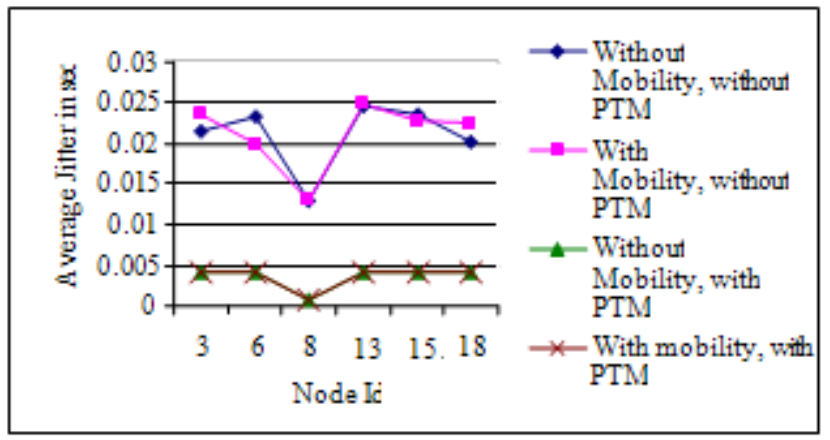

Figure 5.d Average Jitter analysis for Grid Topology

The figure 6.(a-d) shows the performance analysis of 802.15.4 network and other WLAN nodes for Random topology. In this topology heterogeneous nodes are placed randomly. Like other topologies simulation is carried out with and without the random way point mobility model.

The bit error is reduced to zero for the random topology when the PTM scheme is adapted .The figure 6.a witness the results of bit error analysis for random topology. When the proposed scheme is not adapted for transmission the bit error is maximum with the mobility model. The packet transmission is completed with in the simulation time. By default in Qualnet 4.5 simulator simulation time is fixed as 30 s.For each node $1 \mathrm{~s}$ is allotted for packet transmission.

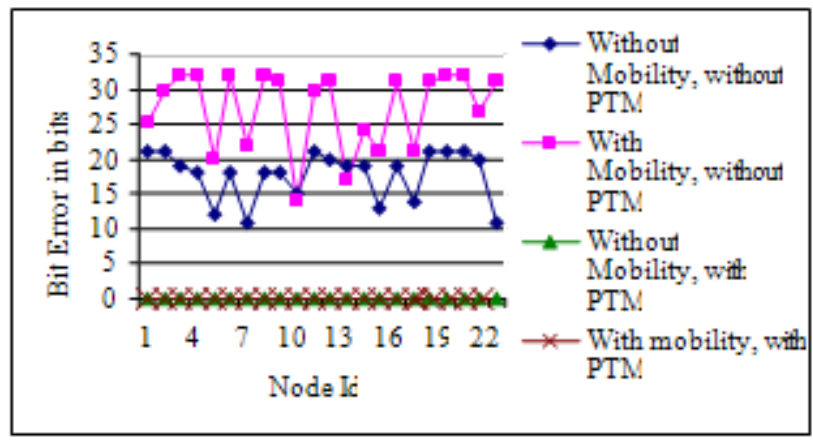

Figure 6.a Bit Error analysis for Random Topology

The figure 6.b shows the throughput analysis for random topology with and without the mobility model. In random topology node 10 and 21 are WPAN nodes and other nodes are WLAN nodes. In real time environment random topology is realized. After allocating the time for packet transmission the throughput is increased by 7 times the throughput measured before implementing the proposed scheme.

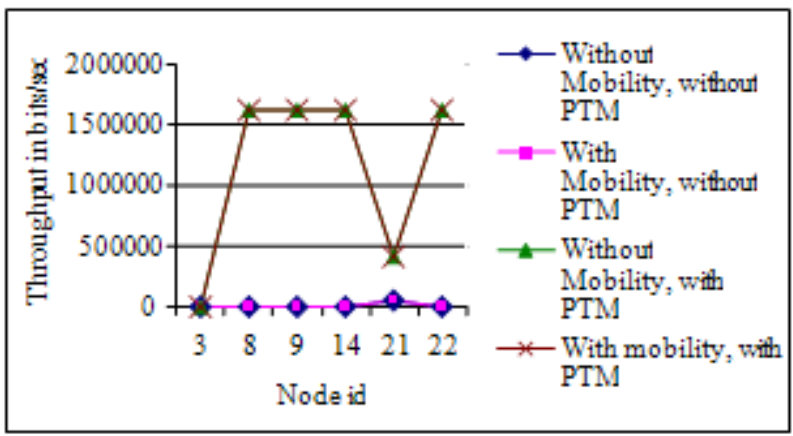

Figure 6.b Throughput analysis for Random Topology

The average end-end delay and average jitter analysis is shown in figure 6 (c-d). The average end-end delay for WPAN node is decreased by 18 and 19.7 times when the nodes are ideal and moving. For WLAN node it is 4 times and 5 times respectively. The average jitter is reduced by 15 times for WPAN node and 5 times reduced for WLAN node.

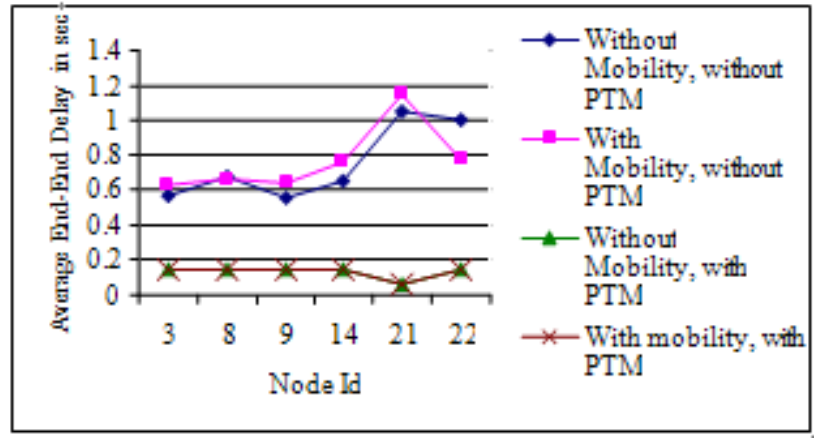

Figure 6.c Average End-End delay analysis for Random Topology

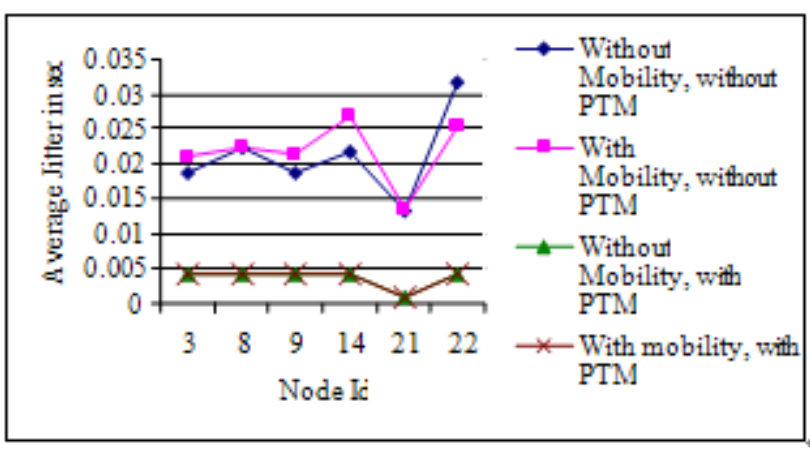

Figure 6.d Average Jitter analysis for Random Topology

\section{CONCLUSION}

We in this paper present analysis on performance of coexistence heterogeneous networks. In this paper, we propose a new scheme using packet transmission management for the coexistence of IEEE 802.15.4 LRWPAN and IEEE 802.11b WLAN.The performance of IEEE 802.15.4 network is analyzed when the nodes are moving randomly. The simulation results show that the proposed scheme is effective in performance improvement for coexistence network of IEEE 802.15.4 for circular, grid and random topologies. In this paper only 25\% WLAN nodes are considered as transmitting nodes. In future the analysis can be extended for grid, circular and random topology with $50 \%$, $75 \%$ and $100 \%$ transmitting nodes. 


\section{REFERENCES}

[1] ZigBee Alliance Web Site, http://www.zigbee.org

[2] IEEE 802 Working Group: Standard for Part 15.4,"Wireless Medium Access Control(MAC) and Physical Layer(PHY) Specifications for Low Rate Wireless Personal Area Networks(LR-WPANs)," ANSI/IEEE 802.15.4, 2003

[3] "Ieee 802.11, the working group setting the standards for wireless lans,"http://grouper.ieee.org/groups/802/11/.

[4] J. Lansford, A. Stephens, and R. Nevo, "Wi-fi (802.11b) and bluetooth:Enabling coexistence," IEEE Network, pp. 20-27, Sept/Oct 2001.

[5] Ling-Jyh Chen, Tony Sun, Mario Gerla," Modeling Channel Conflict Probabilities between IEEE 802.15 based Wireless Personal Area Networks", Communications, 2006. ICC apos;06. IEEE International Conference on Vol.1, Issue, June 2006 Page(s):343 - 348

[6] Soo Young Shin, Hong Seong Parky, Sunghyun Choi, Wook Hyun Kwon," Packet Error Rate Analysis of IEEE 802.15.4 under IEEE 802.11b Interference", IEICE Transactions on Communications 2007

[7] Dae Gil Yoon, Soo Young Shin, Wook Hyun Kwon and Hong Seong Park ," Packet Error Rate Analysis of IEEE 802.11b under IEEE 802.15.4 Interference" Vehicular Technology Conference, 2006. VTC 2006-Spring. IEEE 63rd Publication Date: 7-10 May 2006 Volume: 3, On page(s): 1186-1190

[8] G.M.Tamilselvan, Dr.A.Shanmugam, "Modelling Channel Conflict Probabilities and Interference analysis of Coexistent Heterogeneous Networks" International Journal of Computer science and Knowledge Engineering (IJCSKE), Vol 3.Jan-June 2009,On page(s): 113-118

[9] G.M.Tamilselvan, Dr.A.Shanmugam, "Probability Analysis of Channel Collision between IEEE 802.15.4 and IEEE 802.11b using Qualnet Simulation for Various Topologies" International Journal of Computer Theory and Engineering, Vol. 1, No. 1, April 2009,On page(s):59-64

[10] Ivan Howit and Jose A. Gutierrez, "IEEE 802.15.4 Low RateWireless Personal Area Network Coexistence," Issues Wireless Communications and Networking, Vol.3, pp. 1481-1486, 2003.

[11] Steibeis-Transfer Centre, "Compatibility of IEEE802.15.4 (Zigbee) with IEEE802.11 (WLAN), Bluetooth, and Microwave Ovens in 2.4 GHz ISM-Band," http://www.ba-loerrach.de.

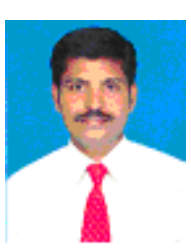

G.M.Tamilselvan received BE Degree in Electronics and Communication Engineering from Tamilnadu College of Engineering, Coimbatore in 1998 and ME Degree in Process Control and Instrumentation from Faculty of Engineering and Technology, Annamalai University in 2004.From 1998 to 2002 he worked as a faculty in NIIT.He was working as Lecturer in the department of ECE, Erode Sengunthar Engineering College during 2004-2007. Currently he is working as Lecturer in the Department of ECE, Bannariamman Institute of Technology, Sathyamangalam.He is doing part time research in Anna University, Coimbatore.His current research focuses on wireless heterogeneous networks, Interference mitigation, Mesh networks and Adhoc networks. He is member of ISTE and IACSIT, Singapore.

E-mail:tamiltamil@rediffmail.com

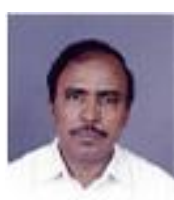

Dr.A.Shanmugam received the BE Degree in PSG College of Technology in 1972, Coimbatore and ME Degree from College of Engineering, Guindy, Chennai in 1978 and Doctor of Philosophy in Electrical Engineering from Bharathiar University, Coimbatore in 1994.From 1972-76, he worked as Testing Engineer in Testing and Development Centre, Chennai. He was working as a Lecturer Annamalai University in 1978. He was the Professor and Head of Electronics and Communication Engineering Department at PSG College of Technology, Coimbatore during 1999 to 2004. Authored a book titled "Computer Communication Networks" which is published by ISTE, New Delhi, 2000.He is currently the Principal, Bannari Amman Institute of Technology, Sathyamangalam.He is on the editorial board of International Journal Artificial Intelligence in Engineering \& Technology (ICAIET), University of Malaysia, International Journal on "Systemics, Cybernetics and Informatics (IJSCI)" Pentagram Research Centre, Hyderabad, India. He is member of the IEEE, the IEEE computer society.

E-mail : dras@yahoo.co.in 